\title{
A traffic micro-simulation model to estimate four-lane highways' capacity in Thailand
}

\author{
Wuttikrai Chaipanha ${ }^{1}$, Ladda Tanwanichkul ${ }^{1, *}$, and Jumrus Pitaksringkarn ${ }^{2,}$ \\ ${ }^{1}$ Department of Civil Engineering, Faculty of Engineering, Khon Kaen University, Khon Kaen, Thailand \\ ${ }^{2}$ Department of Civil Engineering, Faculty of Engineering, King Mongkut's Institute of Technology Ladkrabang, Bangkok, Thailand
}

\begin{abstract}
This article purposed to present the maximum capacity and to develop the equation in the capacity estimation of 4 types of four-lane highways by using the micro-simulation model. Regarding the analysis, the factors affecting the capacity include access-point, heavy vehicles and median u-turn. According to the study, it was found that the maximum capacity of the four-lane highways in type 1 is 2194 passenger car/hour/lane. In the descending orders, the maximum capacity of the highways with four lanes in type 2, 3, and 4 are 2161, 2094 and 2017 passenger car/hour/lane, respectively. At the same time, the maximum capacity of the prevailing condition in the study is $1300-1600$ vehicles/hour/lane, which is different from the HCM2010 method for 20-30\%, due to the Thai's highway characteristics directly affect the traffic and driving behavior. Median u-turn affects the four-lane highways in type 1 and 2 the most, while access-point factor has the most influence towards type 3 and 4 . In addition, the author has developed the equation models for capacity estimation, which the result derived from the relationship between the capacities and affecting factors. It was aimed at using as the guidelines in the capacity assessment of Thai's four-lane highways in the future.
\end{abstract}

\section{Introduction}

Highway capacity is one of the most important applications of traffic theory, with analysis and design of transportation facilities leading to competent decisionmaking to improve road networks [1-3]. The United States Highway Capacity Manual (US-HCM) is regarded as the global standard [4]. However, this was developed specifically for the United States and may not be relevant in other countries which have unique traffic characteristics. Some nations have attempted to modify the US-HCM and develop highway capacity manuals specifically suited to their individual requirements as the Indian, Swedish, Indonesian, Korea and German.

Estimation of the highways' capacity in Thailand was also based on the US-HCM; however, the physical characteristics of Thai highways are different from the USA [5]. Therefore, it is an interesting gap to consider the development of a highway capacity estimation method designed especially for Thai's highways.

Methods of estimating roadway capacity can be divided into two categories as Direct Empirical Methods, based on observed traffic flow characteristics and Indirect Empirical Methods, based on guidelines and simulation models [6]. However, a direct empirical method generally requires a survey to be conducted under capacity conditions, and it also is relatively uncommon for a capacity situation to occur on a regular weekday. A micro-simulation model is often used in indirect empirical analyses to reduce the limitations of the survey and complex phenomena which are difficult to analyze by mathematical techniques. Several previous studies have used traffic micro-simulation models to identify factors affecting roadway capacity [7-12].

This research investigated the application of a microsimulation model to estimate the capacity of four-lane highways in Thailand, which is important and account for about one-third of the total highway distance [13]. Maximum capacity for base and prevailing conditions was identified and equation models were developed to estimate the capacity of four types of four-lane highways. Consider the key factors negatively affecting the capacity included (1) access-points, (2) heavy vehicles, and (3) median u-turn (Fig. 1).

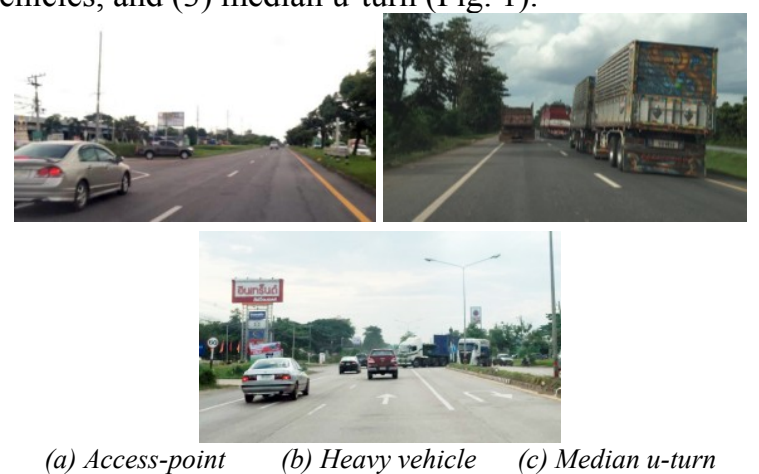

Fig. 1. Factors affecting four-lane highways' capacity in Thailand 


\section{Methodology}

\subsection{Site selection}

Four-lane highways represent multi-lane rural road networks in Thailand and many characteristics and factors differ from the US-HCM analysis conditions. The study was divided into four types of cross-sectional design in accordance with the standard of the Department of Highways [14] as shown in (Table 1).

Table 1. Type of four-lane highways in Thailand

\begin{tabular}{|c|c|l|c|}
\hline $\begin{array}{c}\text { Type } \\
\text { no. }\end{array}$ & \multirow{2}{*}{$\begin{array}{c}\text { Divided/ } \\
\text { Undivided }\end{array}$} & \multicolumn{1}{|c|}{ Median type } & $\begin{array}{c}\text { Median } \\
\text { width (m.) }\end{array}$ \\
\hline 1 & \multirow{2}{*}{ Divided } & Depressed median & varies \\
\cline { 3 - 4 } & & Raised median & 4.6 \\
\hline 2 & \multirow{2}{*}{ Undivided } & Painted median* & 2.0 \\
\cline { 3 - 4 } & & Undivided & - \\
\hline 4 & &
\end{tabular}

*Driving behavior on the four-lane highways with painted median is no differences from type 4 , and was identified for undivided.

Highways in the northeast region of Thailand were examined. The criterion of site selection followed the HCM 2010 base condition and represented the prevailing conditions of four-lane highways in Thailand as follows:

- Consist of physical factors that affect the capacity (access-point and median u-turn) to be used to calibrate the driving behavior in the model.

- Each selected highway type shall be designed in accordance with the standard of the Department of Highways, and the highest traffic volume will be compared to the same type [15].

Based on these conditions, the representative four-lane highways sections were selected (Fig. 2).

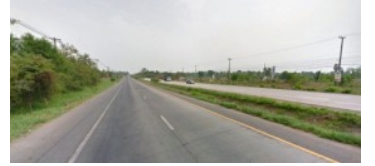

(a) Type1:Hwy.no.2

(Section : Khonkaen-Udonthani) KM.349+000-350+000

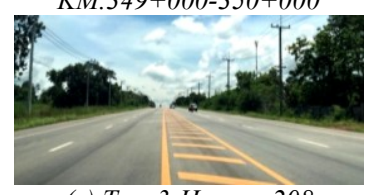

(c) Type3:Hwy.no. 208

(Section : Thaphra-Kosum) KM. $7+000-8+000$

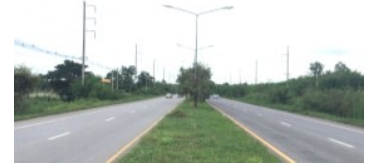

(b) Type2:Hwy.no.12

(Section : Khonkaen-Chumphae) KM.537+000-538+000

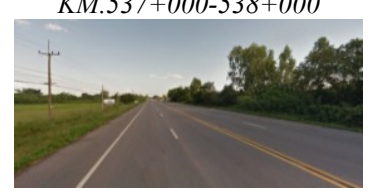

(d) Type4:Hwy.no.208

(Section : Thaphra-Kosum) KM.40+000-41+000
Fig. 2. Selected sections of four-lane highways

\subsection{Data Collection and Preparation}

Data were categorized into four groups as (1) Geometric data: lane width, median width, median type, and aerial photos, (2) Demand data: entry volume, (3) Control data as the speed limits, and (4) Calibration data: spot speed, travel speed, traffic count, and headway.

Entry volume was measured at two intervals of 1 hour 30 minutes each during the morning and evening peak hours. Spot speed surveys followed the desired distributions in the micro-simulation model as a significant variable which affected the capacity [16]. Radar speed gun surveys were conducted during nonpeak hours by vehicle type and traffic lane. In the car following model, the time gap (Headway) that has a determining influence on capacity $[12,16]$. Video data were collected and applied in calibration of the microsimulation model.

\subsection{Base Model Development}

PTV Vissim software was applied to develop a traffic micro-simulation model (Fig. 3.) divided into five steps as (1) Base data for simulation, (2) Traffic networks, (3) Evaluation and configuration, (4) Simulation, and (5) Error checking.
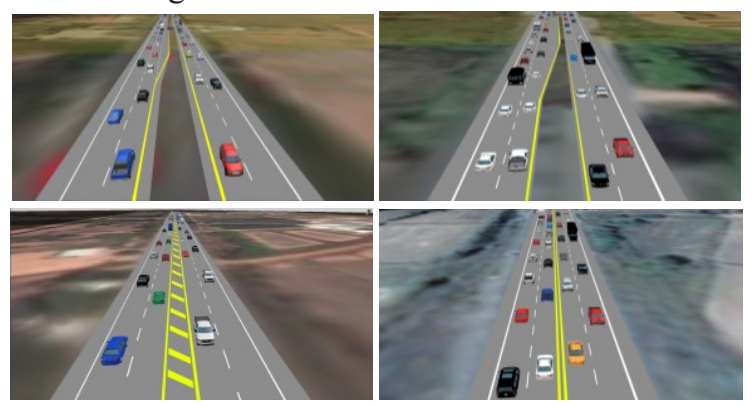

Fig. 3. Example of the base model

\subsection{Model Calibration and Validation}

Traffic volume, and travel speed were used as measures of consistency between the model and local conditions. The calibration and validation target criteria were based on the Design Manual for Roads and Bridges (DMRB) and other acceptable standards [17]. The traffic count comparison has a GEH statistics value of less than 5 and speed of travel not different from the observed over 5\%. In order to calibrate and validate the model, the configuration and adjustment of the values for the VISSIM parameters $\mathrm{CC} 0, \mathrm{CC} 1$ and $\mathrm{CC} 2$ were the most influential variables for cars following model and capacity [18]. From the model calibration and validation, all the GEH values were ranged from 0.1 to 0.4 for the traffic count comparison, while the average percentage differences of the travel speed for each type at 4.2, 0.8 , 1.3 and 2.5 , respectively.

\subsection{Model Application}

The traffic micro-simulation model was applied as a tool to estimate the four-lane highways' capacity, considering various factors including number of access-points per $\mathrm{km}(0,2,4$ and 6), different proportions of heavy vehicles $(0 \%, 5 \%, 15 \%$ and $25 \%)$, and number of median u-turns per $\mathrm{km}(0,1$ and 2) [19].

\subsection{Capacity Estimation}

A calibrated and validated micro-simulation model was used in the capacity estimation, where speed data were estimated under different traffic volumes by increasing 
the flow rate in increments of $500 \mathrm{veh} / \mathrm{hr}$, from an initial value till highway capacity. When the simulated volume reached capacity level, increments in the input traffic volumes did not equate to increase in the exit volume and resulted in a decrease in the rate of traffic flow [20]. Highway capacity estimation was performed using speed-volume relationships by plotting speed on the graph ordinate and volume on the abscissa.

\section{Results and discussion}

\subsection{Estimation of capacity under base condition and prevailing condition}

For the base condition of no access-points, all vehicles as passenger cars, and no median u-turns, the Type 1 fourlane highways had the maximum capacity at 2194 $\mathrm{pc} / \mathrm{hr} / \mathrm{ln}$. With higher physical and standard design, drivers were more likely to travel faster with greater capacity. Four-lane highways Types 2, 3 and 4 recorded 2161, 2094 and $2071 \mathrm{pc} / \mathrm{hr} / \mathrm{ln}$, respectively. All results slightly different from the HCM2010 calculation by $0.4 \%-4.5 \%$ because this was not impacted by any factors.

The traffic micro-simulation model estimated the capacity under prevailing condition (surveyed state) for each type at 1574, 1524, 1433 and $1368 \mathrm{veh} / \mathrm{hr} / \mathrm{ln}$, respectively. The capacity results, which represented the highways in each type, was significantly different from the HCM2010 method due to the factors that affected the capacity to disturb the traffic flow (Table 2).

Table 2. Capacity estimation under prevailing condition compared with the HCM2010 calculation

\begin{tabular}{|c|l|c|c|c|}
\hline \multirow{2}{*}{$\begin{array}{c}\text { Type } \\
\text { no. }\end{array}$} & \multirow{2}{*}{$\begin{array}{c}\text { Median } \\
\text { type }\end{array}$} & \multicolumn{2}{|c|}{$\begin{array}{c}\text { Capacity } \\
\text { (Veh/hr/ln) }\end{array}$} & \multirow{2}{*}{$\begin{array}{c}\text { Diff. } \\
\text { (\%) }\end{array}$} \\
\cline { 3 - 4 } & & $\begin{array}{c}\text { Micro- } \\
\text { Simulation }\end{array}$ & $\begin{array}{c}\text { HCM } \\
\mathbf{2 0 1 0}\end{array}$ & \\
\hline 1 & Depressed median & 1574 & 1963 & -19.8 \\
\hline 2 & Raised median & 1524 & 1992 & -23.5 \\
\hline 3 & Painted median & 1433 & 1999 & -28.3 \\
\hline 4 & Undivided & 1368 & 1991 & -31.3 \\
\hline
\end{tabular}

\subsection{Impact of factors on capacity}

\subsubsection{Impact of access-point on capacity}

Impact of the access-point on four-lane highway capacity for Types 1 and 2 decreased by 1.87 and 1.90\%, respectively, compared to the base condition. However, in the case of four-lane highways for Types 3 and 4, access-points had a significant effect on capacity, which reduced by 47.18 and $46.16 \%$, respectively, compared to the base condition. Access-points involved turning through the traffic resulting in disruption in the flow and reduction in both speed and capacity (Fig. 4).

\subsubsection{Impact of heavy vehicle on capacity}

The impact of heavy vehicles on traffic flow between $0 \%$ and $25 \%$ resulted in a decrease in the capacity of all highways. There was a relatively stable trend of 0.7 $0.8 \%$ decrease in the proportion of heavy vehicles, with a $1 \%$ increase from the base condition. Heavy vehicles need more space between the vehicles in the stream and overtaking or changing lanes require longer distances and cause speed and capacity reduction (Fig. 5).

\subsubsection{Impact of median u-turn on capacity}

Results for four-lane capacity at 0 - 2 points/km for Type 1 and 2 highways showed a decrease in capacity by $26.2 \%$ and $29.5 \%$, respectively, compared to the base condition. Reduced capacity was due to disturbing the traffic in the opposite direction and obstructing the traffic in the same direction as the traffic volume increased (Fig. 6).

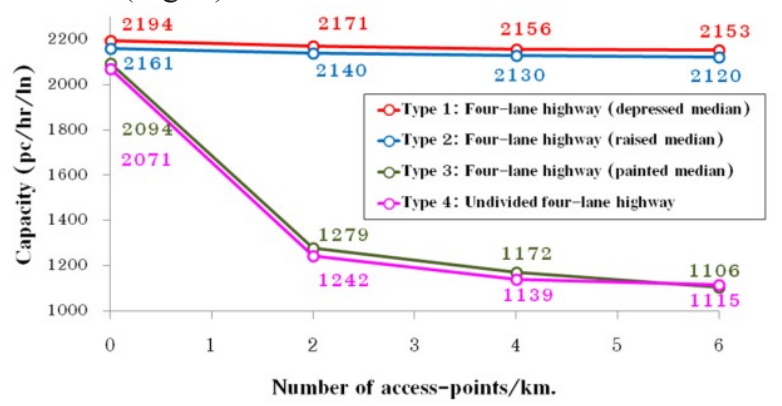

Fig. 4. Impact of access-points on capacity

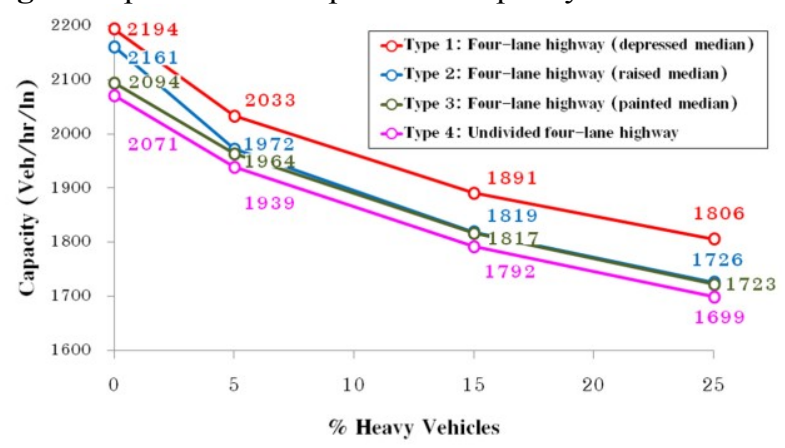

Fig. 5. Impact of heavy vehicles on capacity

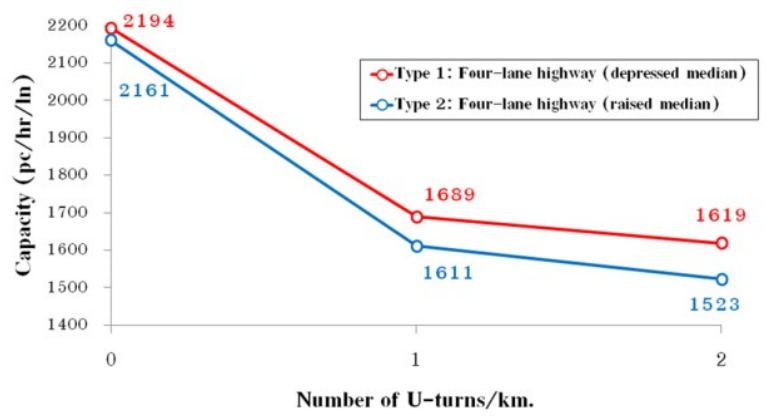

* Types 3 and 4 were assumed as unaffected by median u-turns

Fig. 6. Impact of median u-turns on capacity

\subsection{Capacity model}

The relationships between capacity as a dependent variable and access-point, heavy vehicle, and median u- 
turn as independent variables were investigated. Simple regression analysis was used to check the correlation coefficient (r) between the independent variables, and non-collinear variables are induced into the multiple linear regression models. A multiple linear regression model was generated from the micro-simulation results which estimated four-lane highways' capacity on prevailing conditions as follows:

$$
\begin{aligned}
& C_{\text {Typel }}=2018.466-9.167_{A}-6.932_{H}-211.063_{U} \\
& C_{\text {Type } 2}=2002.649-13.846_{A}-7.977_{H}-262.438_{U} \\
& C_{\text {Type3 }}=1794.303-130.125_{A}-6.749_{H} \\
& C_{\text {Type4 }}=1755.045-123.288_{A}-5.955_{H}
\end{aligned}
$$

Where $\mathrm{C}$ is the capacity for each four-lane highway type $(\mathrm{veh} / \mathrm{hr} / \mathrm{ln}), \mathrm{A}$ is the number of access-points per kilometer (in the range of $0-6$ ), $\mathrm{H}$ is the percentage of heavy vehicles in the traffic stream (in the range of 0 $25 \%$ ), and $U$ is the number of median u-turns per kilometer (in the range of 0-2). From statistical analysis of the model, the coefficients of determination $\left(\mathrm{R}^{2}\right)$ at $0.781,0.875,0.750$ and 0.712 reflected a high goodness of fit. Significance at the $95 \%$ confidence level, with significance of the $F$ statistic at $<0.001$. A negative sign indicated that as the effect of the factors increased, the capacity decreased.

The standardized coefficients $(\beta)$ show the influence of each variable. The median u-turn was the most influential factor for four-lane highways with depressed median (Type 1) and raised median (Type 2). The access-point factor had the most influence toward fourlane highways with painted median (Type 3) and undivided four-lane highways (Type 4).

\section{Conclusion}

The application of a micro-simulation model is another approach to estimate highways' capacity. In addition to reducing the limitations of survey data, micro-simulation models can also effectively emulate local traffic conditions, resulting in more accurate estimations of highways' capacity. In this study, four types of four-lane highways were selected as representative in Thailand; these differed from the conditions of the HCM analysis. Highways with physical and higher design standards have greater capacity due to fewer traffic distractions and higher speeds. However, capacity reduces when affected by various factors and comparisons with the HCM approach were significantly different.

Interactions and conflicts between vehicles interfered with traffic flow on the carriageway and significantly reduced stream speed and capacity. The availability of median u-turns affected four-lane highways with median as Types 1 and 2, while access-points has the highest negative influence on four-lane highways without median as Types 3 and 4. The capacity estimation model can serve as a guideline for transport and traffic operation, and improve design and planning based on the standard of Thailand's Department of Highways (DOH) with assumptions of the level terrain. Further studies should analyze other conditions and roadways that fall under the responsibility of other departments as an interesting approach in the future.

We would like to acknowledge the Department of Civil Engineering, Faculty of Engineering, Khon Kaen University for the research funding.

\section{References}

1. J. Banks, J.S. Carson, B.L.Nelson, D.M. Nicol, Discrete-event system simulation, (2004)

2. V.T. Arasan, R. Koshy, J Transp Eng, 131(7): 544551. (2005)

3. Transport Research Board. Highway capacity manual 2010, (2010)

4. Central Road Research Institute. Development of Indian highway capacity manual, (2014)

5. L. Tanwanichkul, J. Pitaksringkarn, P. Thongkrew. KKU Enj, 39(3):241-248. (2012)

6. M.M. Minderhoud, H. Botma, P.H.L. Bovy. Transp Res Rec, 1572;1997, pp.59-67. (1997)

7. J.H. Kim. A thesis in civil engineering, Pennsylvania state university. (2006)

8. S.S. Arkatkar. IJEE, volume 04, no 06, 2011, pp. 375-380. (2011)

9. Madhu, S. Velmurugan. IJSTER, vol. 1(6), (2011)

10. M.S. Bains, A. Bhardwaj, S. Arkatkar, S. Velmurugan. Procedia Soc Behav Sci, 104. $458-$ 467, (2013)

11. A. Mehar, S. Chandra, S. Velmurugan., KSCE Volume 18, pp 639-645. (2014)

12. S.Chandra, A.Mehar, S.Velmurugan. KSCE, Volume 20, pp 2033-2040. (2015)

13. Department of Highways [Internet]. [cited 2017]. Available from: : http:// maintenance.doh.go.th/website/download/distance2 513-2558.xls. (2014).

14. Department of Highways. Annual Average Daily Traffic on Highways 2015. (2015)

15. Department of Highway. Standard drawings for highway design and construction rev. 2015. (2016)

16. PTV. Vissim user manual. (2011)

17. R. Dowling, A. Skabardonis, V. Alexiadis. Traffic analysis toolbox volume III: guidelines for applying traffic microsimulation modeling software, (2004)

18. G. Gomes, A. May, R. Horowitz. TRR Journal, Vol.1876, pp 71-81, (2004)

19. Department of Highway. Suggestions to consider opening the median of the highway construction. (2005)

20. P.S.Praveen, V.T.Arasan. IJTTE, 3(3): 302 - 330. (2013) 